\title{
Supporting Information: \\ Unraveling the intercalation chemistry of hexagonal tungsten bronze and its optical responses
}

\author{
Yonghyuk Lee, ${ }^{*}$ Taehun Lee, ${ }^{*}$ Woosun Jang, and Aloysius Soon ${ }^{\dagger}$ \\ Global $E^{3}$ Institute and Department of Materials Science and Engineering, \\ Yonsei University, Seoul 120-749, Korea
}

(Dated: June 20, 2016)

\section{ADDITIONAL LINEAR OPTICAL RESPONSE CALCULATIONS (WITH AND WITHOUT INTRABAND DRUDE-LIKE PLASMONIC EXCITATIONS}

Additional linear optical response calculations have been performed using the all-electron full-potential linearized augmented plane wave (FP-LAPW) ELK code[1] within the localdensity approximation (LDA)[2] to the exchange-correlation functional. The augmented plane wave + local orbitals $(\mathrm{APW}+\mathrm{lo})$ basis has been adopted with a maximum length of the reciprocal lattice vector $|\mathbf{G}|$ of $20 \mathrm{a} . \mathrm{u}$. for the expansion of the interstitial potential and density, while the angular momentum is truncated at $l_{\max }=8$ for the expansion of the muffin-tin charge density and potential. The muffin-tin radii $\left(R^{\mathrm{MT}}\right)$ for $\mathrm{W}, \mathrm{O}, \mathrm{K}$, and $\mathrm{Na}$ are set to $2.16,1.39,2.40$, and 2.20 a.u., respectively, and a $|\mathbf{G}+\mathbf{k}|_{\max }$ value of $8 / R_{\mathrm{avg}}^{\mathrm{MT}}$ is enforced for the expansion of the wave functions, where $R_{\mathrm{avg}}^{\mathrm{MT}}$ is the average $R^{\mathrm{MT}}$ listed above for each $h-\mathrm{WO}_{3}$ alloy system. The integration of first irreducible Brillouin zone (IBZ) is performed using a k-point grid of $5 \times 5 \times 7$.

The dielectric responses for the $n$-doped $h$ - $\mathrm{WO}_{3}$ bronze alloys are evaluated with the random phase approximation (RPA) where the electrons and holes are treated independently. To examine the effect of plasmons in the $n$-doped $h$ - $\mathrm{WO}_{3}$, we have included this effect via intraband Drude-like contributions (with a lifetime broadening of $0.001 \mathrm{Ha}$ ) to the dielectric tensor and compared to that without.

Our ELK linear optical response results are then compared with our VASP results (where

\footnotetext{
* These authors contributed equally to this work.

$\dagger$ Corresponding author. E-mail: aloysius.soon@yonsei.ac.kr
} 
indirect and intraband excitations are neglected, as discussed in the main manuscript). We note that for higher photon energy range $(\sim 4$ to $10 \mathrm{eV})$, a noticeable shift in the spectra can be seen in Figs. S1, S2, and S3. We attribute this shift to the use of different exchangecorrelation functionals in the VASP (HSE06) and ELK (LDA) code, and will not change the discussion or conclusions of this work.

(a)

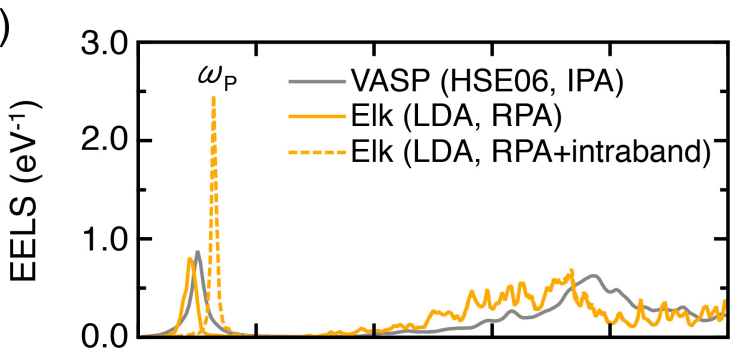

(b)

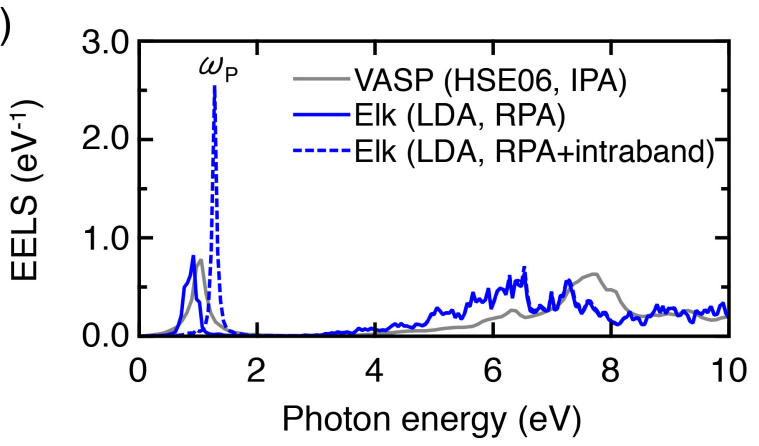

FIG. S1. (Color online) Calculated electron energy-loss spectroscopy (EELS) spectra for (a) $\mathrm{Na}_{0.33} \mathrm{WO}_{3}$, (b) $\mathrm{K}_{0.33} \mathrm{WO}_{3}$. The different computational methods used are labeled according: VASP (HSE06, IPA), ELK (LDA, RPA), and ELK (LDA, RPA including intraband Drude-like contributions). The plasmon frequency peak, $\omega_{\mathrm{p}}$ is labeled. 

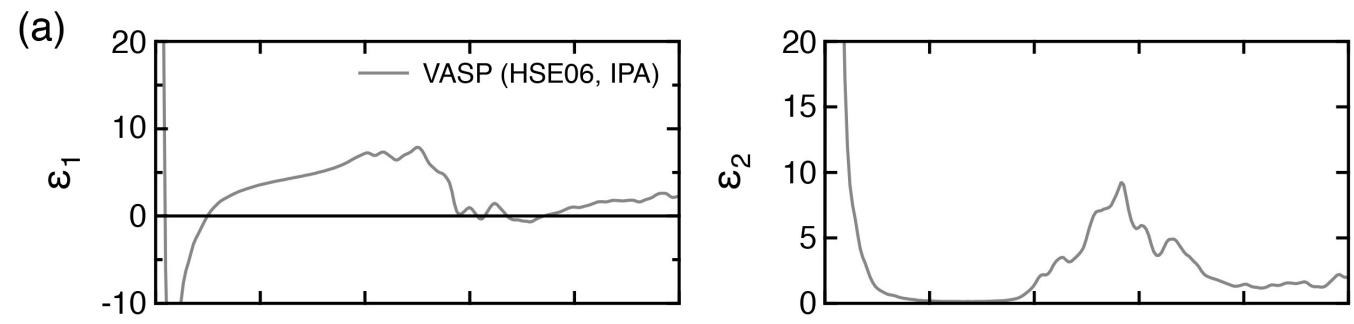

(b)
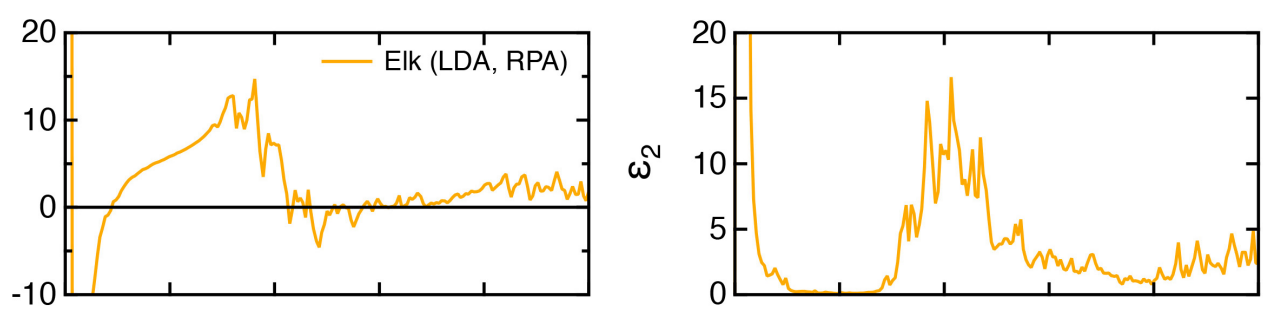

(c)
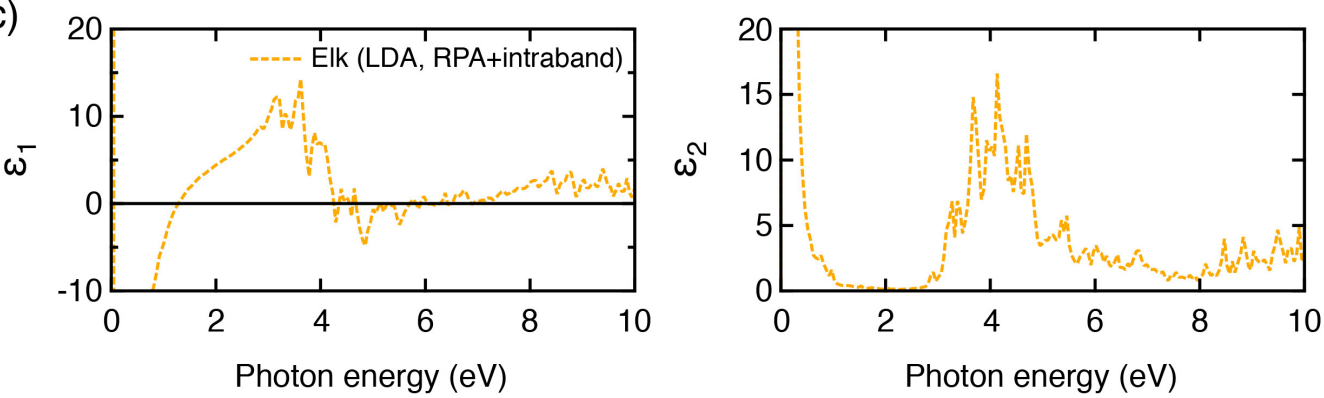

FIG. S2. (Color online) Calculated real $\left(\varepsilon_{1}\right)$ and imaginary $\left(\varepsilon_{2}\right)$ parts of the dielectric function for $\mathrm{Na}_{0.33} \mathrm{WO}_{3}$. The different computational methods used are labeled according: (a) VASP (HSE06, IPA), (b) ELK (LDA, RPA), and (c) ELK (LDA, RPA including intraband Drude-like contributions). 

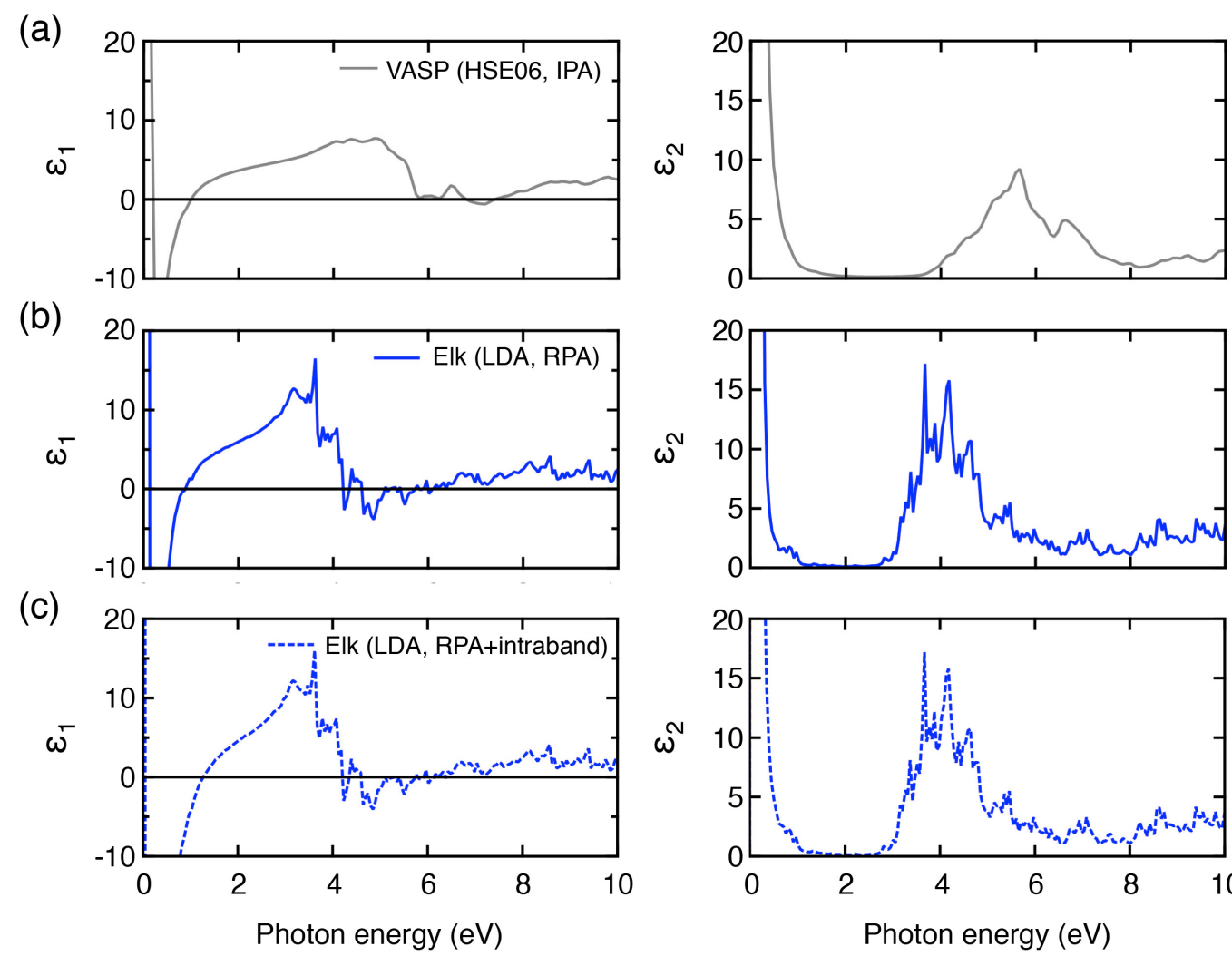

)

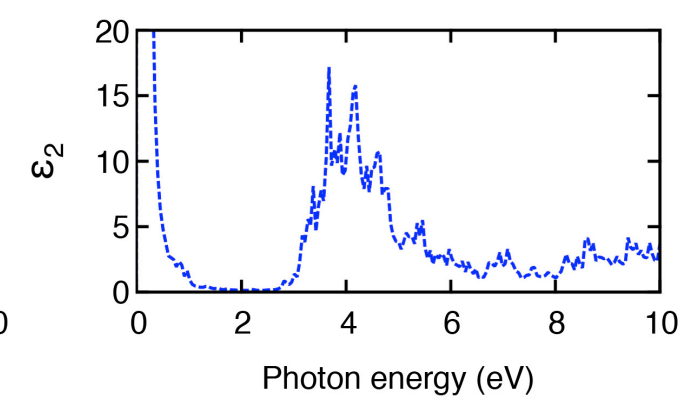

FIG. S3. (Color online) Calculated real $\left(\varepsilon_{1}\right)$ and imaginary $\left(\varepsilon_{2}\right)$ parts of the dielectric function for $\mathrm{K}_{0.33} \mathrm{WO}_{3}$. The different computational methods used are labeled according: (a) VASP (HSE06, IPA), (b) ELK (LDA, RPA), and (c) ELK (LDA, RPA including intraband Drude-like contributions). 
[1] http://elk.sourceforge.net.

[2] J. P. Perdew, Y. Wang. Accurate and simple analytic representation of the electron-gas correlation energy. Phys. Rev. B 1992, 45, 13244. 